\title{
APLICAÇÃO DO MÉTODO SAPEVO-M PARA SELEÇÃO DE UM SISTEMA TMS PARA UMA TRANSPORTADORA
}

\author{
Matheus Dias de Castro \\ Universidade do Grande Rio (UNIGRANRIO) \\ Rua Prof. José de Souza Herdy, 1.160 - Jardim 25 de Agosto, Duque de Caxias/RJ \\ matheus.castro@unigranrio.br \\ Pedro Pimenta Menezes \\ Universidade do Grande Rio (UNIGRANRIO) \\ Rua Prof. José de Souza Herdy, 1.160 - Jardim 25 de Agosto, Duque de Caxias/RJ \\ pp_menezes@hotmail.com \\ Marcos dos Santos \\ Instituto Militar de Engenharia (IME) \\ Praça Gen. Tibúrcio, nº 80, Praia Vermelha, Urca, Rio de Janeiro - RJ \\ marcosdossantos doutorado@yahoo.com.br
}

\section{Luiz Frederico Horácio de Souza de Barros Teixeira}

Centro de Análises de Sistemas Navais (CASNAV)/ Universidade Federal Fluminense (UFF) Praça Barão de Ladário, Ilha das Cobras, Rua da Ponte, Ed. 23, Centro, Rio de Janeiro - RJ frederico.horacio@gmail.com

Carlos Francisco Simões Gomes

Universidade Federal Fluminense (UFF)

Rua Passo da Pátria, 156 - 209, São Domingos, Niterói - RJ

cfsg1@bol.com.br

\section{RESUMO}

O atual ambiente econômico é marcado por um clima de competição acirrada. Tal cenário requer que empresas tenham atenção voltada para a redução de custo e eliminação de desperdícios. A empresa de nome fictício Beta pertence, do ramo de serviços logísticos, é uma transportadora que fornece seus serviços de frota a empresas parceiras. Com o aumento da sua frota devido à grande demanda, as empresas passaram a buscar novas formas de gerenciar o controle de entrada e saída de veículos, visando melhorar sempre a qualidade de seus serviços para atender às necessidades de seus clientes. O objetivo do estudo é selecionar um tipo de sistema TMS (Transport Management System) para otimizar o processo de administração de frota de veículos da transportadora. A metodologia utilizada foi SAPEVOM para determinar uma ordenação entre os critérios através de diferentes tomadores de decisão que avaliaram os critérios. Os resultados obtidos auxiliarão na seleção do melhor sistema a ser implementado pela empresa.

Palavra-chave: Sistema TMS; Gestão de frota; SAPEVO-M. 


\begin{abstract}
The current economic environment is marked by a climate of fierce competition. Such a scenario requires that companies pay attention to reducing costs and eliminating waste. The fictitious company Beta belongs to the Logistics group, it is a carrier that provides its fleet services to partner companies. With the increase in their fleet due to high demand, companies began to look for new ways to manage the control of entry and exit of vehicles, always improving the quality of their services to meet the needs of their customers. The objective of the study is to select a type of TMS (Transport Management System) system to optimize the fleet management process of carrier vehicles. The methodology used was SAPEVO-M to determine an ordering among the criteria through different decision makers who evaluated the criteria. The results obtained will help in the selection of the best system to be implemented by the company.
\end{abstract}

Keywords: TMS system; Fleet management; SAPEVO-M.

\title{
1. INTRODUÇÃO
}

Segundo Simas (2014), os elementos fundamentais para a tomada de decisão sobre escolha de fabricantes e modelos para uma determinada frota e sobre a sua gestão operacional e renovação, são o controle de custo e consumo de fatores médios da frota, bem como o desempenho de cada equipamento individualmente. Dessa maneira, pode-se manter os veículos sempre em bom estado e em operação contínua, portanto, mais rentáveis.

A frota automotiva carrega desde simples encomendas até safras inteiras, abastecendo municípios, contribuindo para o desenvolvimento econômico do País. Desta forma, assim como outras modalidades de transporte, o modal rodoviário necessita de modelos de análise para a seleção dos equipamentos a serem utilizados, bem como para o dimensionamento das frotas que irão suprir às demandas específicas do conteúdo a ser transportado (VALENTE, 2008).

Segundo Ballou (1993), não há função logística dentro de uma organização que possa operar de maneira eficiente sem as necessárias informações de desempenho e custo fornecidas todos os dias.

Tais informações são essenciais para correto planejamento e controle logístico. Um sistema de informação logística (SIL) precisa funcionar de maneira abrangente e possuir uma capacidade suficiente para permitir uma comunicação eficiente entre as áreas funcionais da empresa e os membros do canal de suprimentos” (BALLOU, 1993).

Marques (2002) ressalta as vantagens da aplicação dos Sistemas TMS (Transportation Management System - TMS): diminuição dos gastos com transporte e evolução do nível de serviço ao consumidor; maior rendimento na utilização dos ativos; redução no prazo de planejamento da composição da carga e do plano das entregas; disponibilidade de informações exatas de frete, por consumidor, frota, rota; auxílio ao controle de desempenho.

Dentro deste estudo, o artigo pretende aplicar o método SAPEVO-M para contribuir na escolha de um sistema de gerenciamento de transporte pré-selecionado e conceder outro ponto de vista à decisão. 


\section{DESCRIÇÃO DO PROBLEMA}

A empresa de nome fictício Beta, que atua no ramo de serviços logísticos, é uma transportadora que fornece seus serviços de frota a empresas parceiras.

Em uma última pesquisa realizada pelo FIESP (Federação das Indústrias de São Paulo) no ano de 2016, verificou-se que o modal de transporte mais utilizado pelas empresas ainda é o rodoviário, como mostra a Figura 1.

FIGURA 1 - Matriz de transporte no Brasil.

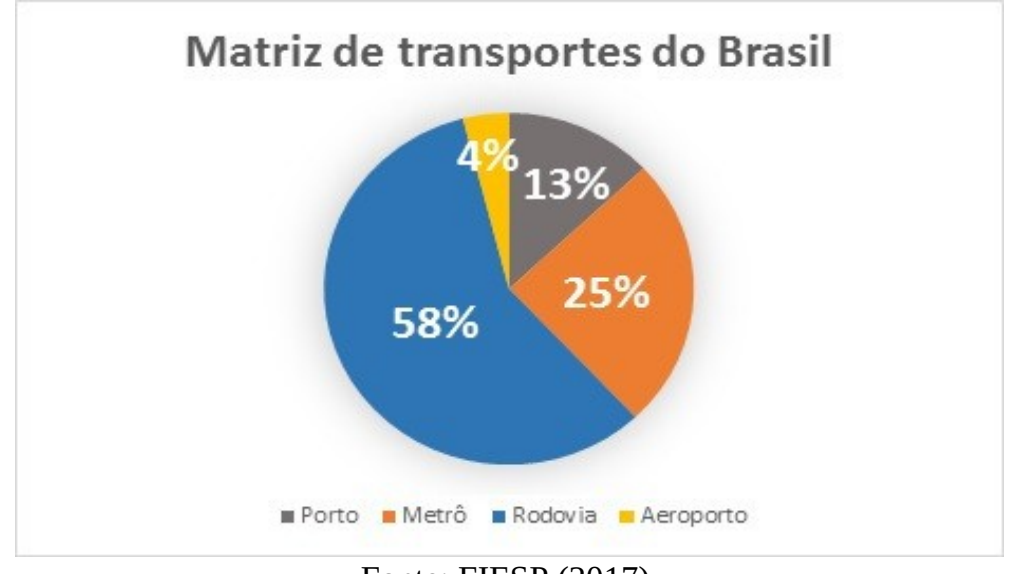

Fonte: FIESP (2017).

O sistema de gestão de frotas é importante para reduzir riscos e prejuízos, além de garantir agilidade. Independentemente do tamanho da empresa e da quantidade de veículos, se não houver um sistema de gestão de frotas, fatalmente o negócio terá problemas. Há diversos fatores que devem ser levados em consideração quando se analisam os veículos: combustível, quilometragem, motoristas, manutenção, rotas, multas, acidentes de trânsito, entre outros (VENY, 2017).

Na era da competição por mercados cada vez mais exigentes, no qual as empresas que possuem êxito no mercado são aquelas que conseguem proporcionar o menor custo das suas entregas ao menor prazo possível, o gerenciamento de frota transforma-se em um desafio que abrange diferentes tarefas, tais como a compra de novos veículos, manutenções (preventiva e corretiva), gerenciamento de clientes, contabilidade etc. tendo como foco a alocação de meios de transporte para satisfazer às demandas dos usuários conforme suas necessidades (IWANOWSKI e DOPPER, 2012). Verifica-se para tanto a importante participação da tecnologia da informação como ferramenta para analisar a informação e recomendar decisões sobre um amplo escopo que atravessa funções e empresas, inclusive os que se relacionam com a gestão de frota da organização (CHOPRA e MEINDL, 2011).

Atualmente, durante o fornecimento dos serviços da empresa estudada, a organização não possui um controle de quais veículos estão em rota através de um sistema TMS, todo esse processo é feito através de uma planilha elaborada pelo gestor diariamente, o que dificulta o gerenciamento da sua frota e a prestação do seu serviço, pois a empresa utiliza diariamente aproximadamente $100 \%$ da sua frota.

Com isso, essa organização está em busca de adquirir um sistema TMS para o controle dos seus veículos. Através de reuniões verificou se quais critérios deveriam entrar em questão para a aquisição desse novo sistema, e quais os graus de prioridades seriam dados aos mesmos.

Como forma das organizações que são transportadoras possuir todo o controle dos veículos de sua frota, optou-se por realizar um estudo nesta Empresa Operadora Multimodal do ramo de logística. A intenção é examinar quais os sistemas TMS pré-definidos por essa 
organização e aplicar uma ferramenta que auxilie na escolha de um deles através de seus critérios já definidos.

Os sistemas TMS selecionados pela organização para aplicação do método multicritério SAPEVO-M foram: Sistema TMS Senior; Sistema TMS Generix e por último o software para gestão de frota Sofit.

Para Banzato (2005, p. 91; apud. Silva, 2009, p. 42), o TMS é um software que funciona para a administração do transporte, que permite ao usuário visualizar e controlar a operação logística. Seus principais benefícios são assegurar a rastreabilidade do pedido e a produtividade em todo o processo de distribuição. Ele também é uma ferramenta que tem por objetivo agregar valor ao serviço de transporte de uma empresa e reduzir o custo da área de logística.

Portanto este estudo pretende averiguar qual seria o sistema ideal que a organização Beta deveria adquirir para o controle de sua frota. A Figura 2 a seguir apresenta um mapa cognitivo com as condições de contorno do problema.

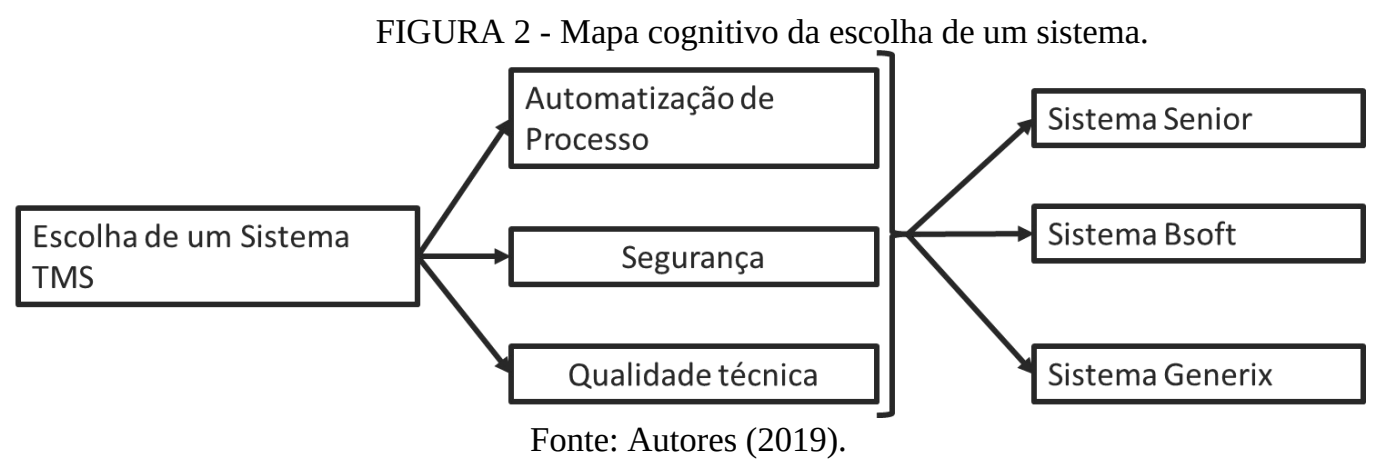

\section{REFERENCIAL TEÓRICO}

\subsection{GESTÃo de Frotas}

Valente et al. (2008) apontam o termo "gestão de frota" como sendo a tarefa de comandar, gerenciar ou conduzir um grupo de veículos referentes a uma mesma empresa. Essa atividade tem uma dimensão gigante e envolve inúmeros serviços, como enquadramento, classificação de equipamentos, roteirização, custos, modernização de veículos, manutenção etc. É um elemento relevante no processo de gestão de transportes, visto que o deslocamento de carga tem uma importância considerável na formação dos custos logísticos e na qualidade do trabalho, já que é a tarefa final da cadeia de suprimentos (BERTAGLIA, 2009).

Para Santos et al. (2012) a fragilidade de um sistema de transporte tem um custo a ser liquidado, pelo atraso que ele proporciona. Um país socialmente avançado sempre tem um sistema qualificado de deslocamento de indivíduos e cargas, não são por coincidência que os países mais desenvolvidos são os que dispõem dos melhores sistemas de transportes, constatando que o nível do PIB está associado com a qualidade dos transportes.

A frota de veículos desloca desde simples cargas até cargas complexas, suprindo municípios, contribuindo no desenvolvimento econômico do País. O transporte rodoviário precisa de esquemas de estudo para seleção de equipamentos a serem usados, assim como para o enquadramento de frotas que irão atender aos requisitos do conteúdo a ser transportado. (VALENTE, 2008)

Um ponto importante na gestão de frotas é a manutenção. Valente (2008) afirma que a manutenção procura manter a frota em boas condições de uso, dentro dos limites econômicos e de forma que a sua imobilização seja mínima. A manutenção é uma atividade 
de importância estratégica para as organizações, devendo garantir a disponibilidade dos equipamentos e instalações com confiabilidade, segurança e custos adequados. Ainda de acordo o autor o tamanho da estrutura dos serviços de manutenção vai depender do número de veículos que compõem a frota e das características da empresa, e que independentemente da composição, preocupação com questões de cuidados diários de manutenção e inspeção dos veículos, por parte dos seus motoristas; manutenção preventiva periódica dos veículos; manutenção corretiva e recuperação de conjuntos e reformas de unidades.

\subsection{SELEÇÃo DOS CRITÉRIOS}

Para a localização de instalações, existem critérios, métodos e modelos utilizados que auxiliam o tomador de decisão na execução de suas atividades. Segundo Yang e Lee (1997), critérios como mercado, transporte, mão de obra, matéria-prima e regulamentação governamental devem ser levados em consideração na hora de decidir onde localizar uma instalação.

Randhawa e West (1995) aponta critérios como minimização de custos, minimização de distâncias, impostos locais, exigências legais, atitudes da comunidade e condições de vida como importantes para localização de instalações.

Chuang (2001) identifica como critérios quantitativos importantes para a localização de instalações os custos do terreno e imóveis, custos de transporte de entrada e saída, quantidade de abastecimento de suprimentos, proximidade com consumidores e varejistas, disponibilidade de mão de obra técnica necessária e, como critérios qualitativos, os fatores ambientais, a qualidade de vida e as políticas do governo.

Moreira (1996) destaca a diferença nos critérios para localização de unidades de prestação de serviço e instalações industriais, sendo proximidade com mercado consumidor, tráfego, e localização dos concorrentes critérios para o primeiro caso e matéria-prima, água, energia e mão de obra os critérios para o segundo caso.

\subsection{SISTEMA TMS (TRANSPORT MANAGEMENT SYSTEM)}

Hughes e Perrons (2011) e Stouthuysen, Slabbinck e Roodhooft (2012), reconhecem a competência que o TMS tem de relacionar elementos internos e externos, que exigem controle. Desta maneira, o software tem como propósito ajudar boa parte das decisões do cotidiano das operações logísticas, com a finalidade de alcançar a otimização na administração da empresa.

Para Rohr (2013), a aplicação da TI, através do TMS, possibilita aos gestores um aumento de agilidade e precisão, ajudando na realização de uma gestão mais competente por meio de uma melhoria na movimentação de recursos envolvidos no planejamento de transportes.

Segundo Gomes e Ribeiro (2014), na integração entre logística e TI, a internet possibilita a troca de informações em tempo real, entre fornecedores e clientes. Desta forma, problemas inesperados são notificados prontamente, de modo a não comprometer a entrega.

Em um sistema para gerenciamento da cadeia de suprimento, as decisões no nível estratégico envolvem todas as etapas de suprimento, produção e fornecimento. Os critérios operacionais auxiliam na hora de tomar as decisões que são embasadas em informações transacionais, com um nível maior de detalhamento, dando suporte ao comando gerencial das operações. O TMS estabelece um padrão de um sistema de gerenciamento da cadeia de suprimento, contribuindo com às decisões no nível operacional, como apresentado na Figura 3. Ou seja, o TMS auxilia a gestão da cadeia de suprimento, nos níveis tático e operacional (MARIA, 2010). 
FIGURA 3 - Gestão da Cadeia de suprimentos.

\begin{tabular}{|c|c|c|c|c|}
\cline { 2 - 4 } Estratégico & \multicolumn{3}{|c|}{ Planejamento da Rede (Newtork Planning) } \\
\cline { 2 - 5 } Tático & \multicolumn{3}{|c|}{ Planejamento Mestre da Distribuição } & Planejamento do \\
\cline { 2 - 5 } Operacional & $\begin{array}{c}\text { Atendimento à } \\
\text { Demanda }\end{array}$ \\
\cline { 2 - 5 } & $\begin{array}{c}\text { Suprimento de Estoques e de Suprimento } \\
\text { SRM }\end{array}$ & $\begin{array}{c}\text { Planejamento das } \\
\text { Operações }\end{array}$ & $\begin{array}{c}\text { Serviço de } \\
\text { Distribuição CRM }\end{array}$ & TMW / WMS \\
\cline { 2 - 5 } & &
\end{tabular}

Fonte: Maria (2010).

\subsection{O MÉTODo SAPEVO-M}

Criado por Gomes, Mury e Gomes (1997), O método Simple Aggregation of Preferences Expressed by Ordinal Vectors (SAPEVO) consiste, basicamente, em dois processos:

1. Transformação ordinal da preferência entre critérios, expressada por um vetor representando os pesos dos critérios;

2. Transformação ordinal da preferência entre alternativas dentro de um determinado conjunto de critérios. Ao serem agregadas as preferências para todos os critérios, neste segundo processo, é gerada uma matriz de avaliação.

O método SAPEVO-M (Simple Aggregation of Preferences Expressed by Ordinal Vectors -Multi Decision Makers) representa uma nova versão do método ordinal original SAPEVO que possibilitava a avaliação de apenas um decisor. Esta versão evoluída estende o método a múltiplos decisores, além de introduzir um processo de normalização das matrizes de avaliação, incrementando a consistência do modelo (TEIXEIRA et al., 2019).

As informações de preferência no SAPEVO-M são denotadas por uma série de comparações pareadas entre as alternativas. A relação entre as alternativas é expressa em uma escala de sete pontos, na qual são mensuradas, relativamente, a importância entre as alternativas. A partir da avaliação entre alternativas, é obtida uma matriz com a representação numérica correspondente. O resultado da preferência entre as alternativas é expresso pelo vetor resultante da multiplicação matricial entre o vetor peso dos critérios $\mathrm{V}$ e a matriz de avaliação das alternativas $M$. As alternativas são, então, ordenadas em ordem decrescente dos valores numéricos obtidos, gerando-se o ranking desejado. (TEIXEIRA et al., 2019).

\section{PROPOSTA DE SOLUÇÃO}

\subsection{LEVANTAMENTO DE ALTERNATIVAS VIÁVEIS}

Para o desenvolvimento do trabalho foram selecionados três tipos de sistemas, que são detalhados a seguir.

\section{SISTEMA TMS SENIOR}

- Agilidade e automatização na emissão de CT-es;

- Controle da eficiência das entregas;

- Gestão dos volumes transportados com total rastreabilidade; 
- Portal completo do embarcador, possibilitando a solicitação de coleta, baixa de comprovantes de entrega, documentos de transporte e faturas de cobrança;

- Negociações e tabelas de preço atendendo todo tipo de transporte, tanto para pagamento quanto faturamento;

- Comunicação e integração com gerenciadoras de risco;

- Averbação eletrônica das cargas;

- Análise de rentabilidade do frete.

\section{SISTEMA TMS BSOFT}

- Emissão de Conhecimento de Transporte Eletrônico (CT-e);

- Manifesto Eletrônico de Documentos (MDF-e);

- Recibo de Pagamento Autônomo (RPA);

- Controle de faturamento;

- Auditoria e controle de frete;

- Controle de manutenções;

- Monitoramento de pneus.

\section{SISTEMA TMS GENERIX}

- Agendamento de Frete;

- Otimização de Rotas;

- Previsões e Faturamento de transporte;

- Gestão de documentos;

- Controle e medição de performance;

- Comprometimento eco responsável.

\subsection{CRItÉRIOS Utilizados}

Com os tipos de sistemas apresentados, os critérios a serem avaliados para a evolução do trabalho são explicados abaixo:

- QUALIDADE TÉCNICA: Ter amplo acesso à informação de qualidade, de forma fácil, porém customizável, permite ao gestor tomar decisões bem informadas mais rapidamente para que assim possa realizar um planejamento estratégico melhor.

- SEGURANÇA: neste ponto avalia que os dados estarão seguros no sistema em nuvem, através de senhas complexas e diferentes meios de acesso.

- AUTOMATIZAÇÃO DE PROCESSOS: considera se também como este sistema consegue relacionar a troca de informações entre todos os departamentos da empresa.

\subsection{Modelo Matemático}

Para começar o desenvolvimento do trabalho foram entrevistados três funcionários de importantes cargo na empresa. O supervisor de Frota, O gerente de Frota e o analista de sistemas. Para começar com o desenvolvimento do modelo, é apresentada a tabela com a escala de critérios utilizada, Figura 4 a seguir.

\section{FIGURA 4 - Escala de critérios.}




\begin{tabular}{|l|l|l|}
\hline Escala 1 (símbolo) & Escala 1 (variável /expressão Linguística Correspondente) & Escala 2 \\
\hline$<<\ll 1 ~$ & Absolutamente pior / Absolutamente menos importante & -3 \\
\hline$<\ll 1$ & Muito pior / Muito menos importante & -2 \\
\hline$\ll 1$ & Pior / Menos importante & -1 \\
\hline 1 & Igual ou equivalente / Tão importante quanto & 0 \\
\hline$\| 1$ & Melhor / Mais importante & 1 \\
\hline$>» 1$ & Muito melhor / Muito mais importante & 2 \\
\hline$>\gg » 1$ & Absolutamente melhor / Absolutamente mais importante & 3 \\
\hline \multicolumn{2}{|l}{ Fonte: Teixeira, Santos e Gomes (2018). } \\
\end{tabular}

Depois de conhecer os critérios que serão avaliados e suas escalas, os tomadores de decisão da organização expressam suas opiniões sobre os critérios em ordem de preferência gerando assim um "peso" para cada critério, Figura 5 a seguir.

FIGURA 5 - Vetor peso dos critérios.

\begin{tabular}{|c|c|c|c|}
\hline $\begin{array}{l}\text { (DM1C1) } \\
\text { QUALIDADE } \\
\text { TÉCNICA } \\
\end{array}$ & $\begin{array}{l}\text { (DM2C1) } \\
\text { QUALIDADE } \\
\text { TÉCNICA } \\
\end{array}$ & $\begin{array}{c}\text { (DM3C1) } \\
\text { QUALIDADE } \\
\text { TÉCNICA } \\
\end{array}$ & PESO C1 (Soma) \\
\hline 1 & 0,95 & 1,05 & 3,0 \\
\hline (DM1C2) SEGURANÇA & (DM2C2) SEGURANÇA & (DM3C2) SEGURANÇA & PESO C2 (Soma) \\
\hline 0,005 & 0,5 & 0,053 & 0,558 \\
\hline $\begin{array}{c}\text { (DM1C3) } \\
\text { AUTOMATIZAÇÃ } \\
\text { O DE PROCESSOS }\end{array}$ & $\begin{array}{c}\text { (DM2C3) } \\
\text { AUTOMATIZAÇÃ } \\
\text { O DE PROCESSOS }\end{array}$ & $\begin{array}{c}\text { (DM3C3) } \\
\text { AUTOMATIZAÇÃ } \\
\text { O DE PROCESSOS }\end{array}$ & PESO C3 (Soma) \\
\hline 0,5 & 0,05 & 1 & 1,55 \\
\hline
\end{tabular}

Fonte: Autores (2019).

Percebe se que o maior peso foi dado à qualidade técnica, considerado este o critério mais importante na escolha de um sistema TMS.

Portanto, para que se possa obter a matriz de avaliação, cada tomador de decisão irá decidir qual é a sua preferência entre os sistemas com base em cada um dos critérios separadamente. Com isso irá gerar três tabelas por cada um do tomador de decisão que serão agrupadas por critério, com cada uma delas tendo um total. Assim poderá se obter a matriz de avaliação, onde reúne todos os dados dos critérios para cada sistema, Figura 6 a seguir.

FIGURA 6 - Avaliação dos critérios.

\begin{tabular}{|c|c|c|c|}
\hline GERENTE & $\begin{array}{c}\text { GENERIX VS } \\
\text { BSOFT }\end{array}$ & GENERIX VS SENIOR & BSOFT VS SENIOR \\
\hline $\begin{array}{c}\text { QUALIDADE } \\
\text { TÉCNICA }\end{array}$ & 1 & 0 & -1 \\
\hline SEGURANÇA & 2 & 1 & 0 \\
\hline AUTOMATIZAÇÃO & -1 & 1 & 1 \\
\hline
\end{tabular}

\begin{tabular}{|c|c|c|c|}
\hline SUPERVISOR & $\begin{array}{c}\text { GENERIX VS } \\
\text { BSOFT }\end{array}$ & GENERIX VS SENIOR & BSOFT VS SENIOR \\
\hline $\begin{array}{c}\text { QUALIDADE } \\
\text { TÉCNICA }\end{array}$ & 0 & 1 & 1 \\
\hline SEGURANÇA & 3 & -1 & 2 \\
\hline AUTOMATIZAÇÃO & -1 & 2 & 1 \\
\hline
\end{tabular}




\begin{tabular}{|c|c|c|c|}
\hline GERENTE & $\begin{array}{c}\text { GENERIX VS } \\
\text { BSOFT }\end{array}$ & GENERIX VS SENIOR & BSOFT VS SENIOR \\
\hline $\begin{array}{c}\text { QUALIDADE } \\
\text { TÉCNICA }\end{array}$ & -1 & 3 & 1 \\
\hline SEGURANÇA & 2 & 2 & 0 \\
\hline AUTOMATIZAÇÃO & 3 & 1 & -1 \\
\hline
\end{tabular}

Fonte: Autores (2019).

\section{RESULTADOS ALCANÇADOS}

Para a aplicação do método SAPEVO-M foi utilizada a plataforma online gratuita SapevoWeb, disponível no endereço www.sapevoweb.com. O sistema SapevoWeb foi desenvolvido pelo Laboratório de Estudos de Governança, Gestão e Otimização (LEGGO), a partir de uma parceria entre o corpo técnico do Centro de Análise de Sistemas Navais (CASNAV), um grupo de pesquisa do Programa de Pós-Graduação em Engenharia de Produção da Universidade Federal Fluminense (UFF) e um grupo de pesquisa do Programa de Pós-Graduação em Engenharia de Sistemas e Computação do Instituto Militar de Engenharia (IME).

Após a aplicação do método SAPEVO-M por meio da ferramenta SapevoWeb, obteve-se a ordenação global das alternativas, conforme apresentado na Figura 7. Com os resultados obtidos a partir da análise dos critérios, das alternativas e do juízo dos decisores, foi possível verificar que o sistema TMS que apresentou um melhor resultado foi o Sistema Generix, seguido pelo Sistema Bsoft e, em terceiro lugar o Sistema Sênior.

\section{Resultado | Projeto Sistema TMS}

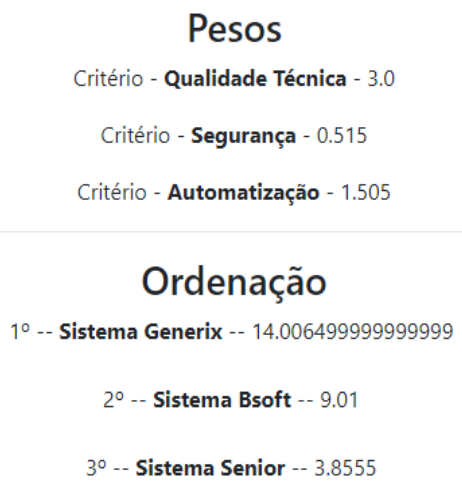

FIGURA 7 - Resultado do projeto TMS. Fonte: Autores (2019).

A partir dos resultados apresentados percebe-se uma significativa diferença nos valores de um sistema para o outro o que expressa claramente que o Generix supera todos os outros sistemas dentro dos critérios elencados qualidade técnica, segurança e automatização. Ele é o sistema que atenderá satisfatoriamente e otimizará o processo de administração de frota de veículos da transportadora.

\section{CONSIDERAÇÕES FINAIS}

Considerando a proposta levantada no artigo em questão, pode se afirmar que os objetivos pré-definidos foram concluídos, visto que se pode obter através da ferramenta SAPEVO-M o sistema adequado para a organização de acordo com a avaliação dos 
indivíduos entrevistados.

A gestão de frotas é uma condição fundamental ao gerenciamento de veículos nas empresas, uma vez que centralizam em seu âmbito todas as atividades de controle (pneus, combustível/abastecimento, análise de quilometragem, manutenção, entre outros) e administração de veículos. Portanto ter um gerenciamento de veículos eficiente significa entender como os elementos de uma frota influenciam na qualidade do serviço de transporte de cargas e é através dessa forma que é possível identificar como estes fatores influenciam o desempenho da organização e como é possível otimizar os seus lucros.

\section{REFERÊNCIAS BIBLIOGRÁFICAS}

[1] BALLOU, Ronald H. Logística empresarial, transportes, administração de materiais e distribuição física. 1.ed. São Paulo: Atlas, 1993.

[2] BANZATO, E. Tecnologia da informação aplicada à logística. São Paulo: IMAM, 2005.

[3] BERTAGLIA, P. R. Logística e gerenciamento da cadeia de abastecimento. 2. Ed.São Paulo: Saraiva, 2009.

[4] CHOPRA, S. MEINDL. P. (2011). Gestão da cadeia de suprimentos: Estratégia, planejamento e operações.4. ed. São Paulo: Pearson Prentice Hall.

[5] CHUANG, P. T. (2001). Combining the analytic hierarchy process and quality function deployment for a location decision from a requirement perspective.

International Journal of Advanced Manufacturing Technology, 18(11), 842-849. http://dx.doi.org/10.1007/s001700170010.

[6] DOPPERS, F. A. IWANOWSKI, S. (2012). E-mobility Fleet Management Using Ant Algorithms. Social and Behavioral Sciences. n. 54, p. 1058-1067.

[7] FIESP - FEDERAÇÃO DAS INDÚSTRIAS DO ESTADO DE SÃO PAULO. Conceito de logística. Brasília: Fiesp, 2016. Disponível em: http://www.fiesp.com.br /. Acesso em: 20 ago. 2018.

[8] GOMES, C. F. S.; RIBEIRO, P. C. C. Gestão da Cadeia de Suprimentos integrada à Tecnologia da Informação, 2ª ed. Rio de Janeiro: Editora SENAC, 2014.

[9] GOMES, L. F. A. M., MURY, A. R., GOMES, C. F. S. (1997). Multicriteria Ranking with Ordinal Data. SAMS, vol. 27, pp. 139 - 145.

[10] HUGHES, Mathew; PERRONS, Robert K. Shaping and re-shaping social capital in buyer-supplier relationships. Journal of Business Research, v. 64, p. 164-171, 2011.

[11] MOREIRA, D. A. (1996). Administração da produção e operações (2 ed.). São Paulo: Pioneira.

[12] RANDHAWA, S. U., \& WEST, T. M. (1995). An integrated approach to facility location problems. Computers \& Industrial Engineering, 29(1-4), 261-265. http://dx.doi. org/10.1016/0360-8352(95)00082-C.

[13] ROHR, M.P. Ferramenta otimizadora e roteirização de cargas. 2013. Monografia Centro de Ciências Exatas e Tecnológicas da UNIVATES, Lajeado, 2013.

[14] SANTOS, Greyciane P. D., et al. (2012). Os benefícios da utilização de indicadores de desempenho na gestão de frota para controle de custos logísticos de transporte $-\mathrm{O}$ 
caso de uma indústria de alimentos. Anais do XXXII Encontro Nacional de Engenharia de Produção, Bento Gonçalves-RS: 2012.

[15] SIMAS, V. (2014). A importância da gestão de frotas para a logística e os negócios. Disponível em: https://www.revistamundologistica.com.br/artigos/aimportancia-da-gestao-de-frotas-para-a-logistica-e-os-negocios. Acesso em: $10 \mathrm{dez}$. 2018.

[16] STOUTHUYSEN, K.; SLABBINCK, H.; ROODHOOFT, F. Controls, service type and perceived supplier performance in interfirm service exchanges. Journal of Operations Management, v. 30, 2012, p. 423-435.

[17] TEIXEIRA, L. F. H. S. B., SANTOS, M. e GOMES, C. F. S. SapevoWeb Software (v.1). (2018). Disponível em: http://www.sapevoweb.com. Acesso em: 30/08/ 2019.

[18] TEIXEIRA, Luiz Frederico Horácio de Souza de Barros; SANTOS, Marcos dos; GOMES, Carlos Francisco Simões. Proposta e implementação em python do método Simple Aggregation of Preferences Expressed by Ordinal Vectors - Multi Decision Makers: uma ferramenta web simples e intuitiva para Apoio à Decisão Multicritério. In: SIMPÓSIO DE PESQUISA OPERACIONAL E LOGÍSTICA DA MARINHA, 19., 2019, Rio de Janeiro, RJ. Anais [...]. Rio de Janeiro: Centro de Análises de Sistemas Navais, 2019.

[19] VALENTE, A. M. Qualidade e produtividade nos transportes. São Paulo: Editora Cengange Learning, $2^{\mathrm{a}}$ Edição Revista, 2008.

[20] VENY. L. C. (2017). Qual a importância do sistema de gestão de frotas. Disponível em: https://www.quatenusonline.com.br/blog/importancia-do-sistema-de-gestao-defrotas/. Acesso em: 2 de ago. 2018.

[21] YANG, J., \& LEE, H. (1997). An AHP decision model for facility location selection. Facilities, 15(Iss: 9), 241- 254. http://dx.doi.org/10.1108/02632779710178785. 